\title{
Los Big Five y el Efecto Moderador de la Resistencia en el Agotamiento Emocional
}

\section{Big Five Factors and Resiliency Moderator Effect on Emotional Exhaustion}

\author{
Antonio León García-Izquierdo \\ Universidad de Oviedo \\ Pedro José Ramos-Villagrasa \\ Universidad de Barcelona \\ Mariano García-Izquierdo \\ Universidad de Murcia
}

\begin{abstract}
Resumen. El objetivo del presente estudio es analizar las relaciones entre los Big Five y la resistencia con el agotamiento emocional en una muestra multiocupacional. La muestra está compuesta por 311 trabajadores de diferentes sectores, aunque predominan los pertenecientes al sector servicios. Se les aplicaron los cuestionarios MBI, BFI y CD-RISC, mediante un diseño transversal. Tal como esperábamos por estudios anteriores hemos encontrado asociaciones significativas entre las variables seleccionadas. Mediante regresión múltiple obtuvimos la capacidad predictiva de los factores de personalidad Neuroticismo, Conciencia y Resistencia. Asimismo, en nuestro estudio hemos encontrado que la resistencia actúa como moderador entre la personalidad y el agotamiento emocional, ya que la resistencia presenta interacción con el factor de personalidad Conciencia. De esta manera, se pone de manifiesto la importancia de los factores de personalidad en la dimensión fundamental del burnout, el agotamiento emocional; así como de la resistencia, una variable poco estudiada que se presenta prometedora en el contexto laboral. La relevancia de tales asociaciones se incrementa al ser escasos los estudios que han abordado esta problemática.

Palabras clave: big five, burnout, agotamiento emocional, resistencia, interacción.
\end{abstract}

\begin{abstract}
The aim of this study is to analyze the relationship between personality (Big Five) and resilience, with emotional exhaustion in a diverse sample. The sample is 311 workers from different sectors, but predominantly within the service sector. We use MBI, BFI and CD-RISC, as instruments in a cross-sectional design study. As expected by previous studies, we have found significant associations between selected variables.. Moreover, we found that resilience acted as a moderator variable between personality and emotional exhaustion, as results has shown a significant interaction between Resilience and Conscientiousness. By multiple regression analysis, we obtained the predictive capability of the personality factor Neuroticism, Resilience, and the product of Conscientiousness and Resilience. Consequently, it highlights the importance of personality in the core dimension of burnout, emotional exhaustion. Further, resilience is a promising variable to be considered in organizational and employment studies. This is relevance as few studies have addressed this problem.

Key words: big five, burnout, emotional exhaustion, resilience, interaction.
\end{abstract}

Vivimos una época donde el cambio y las turbulencias del entorno forman parte consustancial de las dinámicas organizacionales. Asistimos a un nuevo escenario en el que la flexibilidad organizacional presenta a los trabajadores mayores exigencias de adaptación a nuevas situaciones y un aumento de la incertidumbre (Sparks, Faragher y Cooper, 2001). Estos

\footnotetext{
Antonio León García Izquierdo, Departamento de Psicología, Universidad de Oviedo, Pedro José Ramos Villagrasa, Departamento de Psicología Social, Universidad de Barcelona, Mariano García Izquierdo, Departamento de Psicología Social, Universidad de Murcia. Este artículo es producto de la financiación del proyecto de investigación conjunto de la Fundación MAPFRE y la Universidad de Oviedo (FUO-EM-039-06). Los autores quieren agradecer el esfuerzo y la contribución de los revisores y los editores de Revista de Psicología del Trabajo y de las Organizaciones, que han mejorado sustancialmente el manuscrito original. La correspondencia sobre este artículo se enviará al primer autor al Departamento de psicología, Facultad de Psicología, Universidad de Oviedo, Plaza Feijoó, s/n, 33003 Oviedo (España). Email: angarcia@uniovi.es
}

esfuerzos de adaptación tienen efectos adversos en la salud, las actitudes y la calidad de vida laboral de los trabajadores (García-Izquierdo y Ramos-Villagrasa, 2009). En suma, en un escenario dominado por el estrés laboral, las capacidades psicológicas personales cobran una importancia vital (Luthans, Vogelgesang y Lester, 2006). De hecho, Beddington et al. (2008) destacan como una de las conclusiones más relevantes del estudio Foresight Project on Mental Capital and Wellbeing, que en un entorno laboral cambiante el bienestar de los trabajadores es un factor de gran importancia para mejorar el conocimiento en la economía y en la sociedad. Entre los factores más sobresalientes, destaca el burnout (síndrome de quemarse por el trabajo o de desgaste profesional), que puede entenderse como una respuesta al estrés laboral crónico compuesta por agotamiento emocional, despersonalización y reducción de los logros personales (Maslach, 1993). El agotamiento emocional se refiere a los sentimientos de estar emocionalmente consumido; la des- 
personalización es una respuesta insensible del trabajador hacia el usuario de los servicios que presta, y el tercer síntoma, realización personal, trata de la percepción negativa de competencia en el trabajo. Inicialmente, Maslach (1982) identificó las llamadas profesiones de ayuda (helping professsions) como aquellas donde el burnout podía tener especial incidencia, pero más tarde el concepto se aplicó a otras profesiones (Golembiewski, Boudreau, Munzenreider y Luo, 1996; Schaufeli, Leiter, Maslach y Jackson, 1996) señalándose tres dimensiones similares a las citadas: agotamiento, cinismo y eficacia profesional. Aunque se repite la estructura tridimensional (Schutte, Toppinen, Kalimo y Schaufeli, 2000), suele haber consenso en considerar que el agotamiento emocional es la dimensión clave, el elemento fundamental del síndrome de burnout (Lee y Asforth, 1996; Maslach, Schaufeli y Leiter, 2001; Shirom, 1989). Además, permite distinguir el burnout de otros conceptos que pueden estar teóricamente relacionados, como la autoeficacia (Shirom, 1989).

Una constante en la investigación sobre burnout ha sido la búsqueda de consecuentes y antecedentes. En cuanto a las primeros, destacan los efectos físicos y psicológicos en los trabajadores, así como el aumento en la rotación externa, el absentismo, la intención de abandonar la organización, la expresión de actitudes negativas hacia el trabajo, el compromiso con la organización y el descenso en los niveles de rendimiento (Burke y Greenglass, 1996; Cropanzano, 1998; Cropanzano, Ruppe y Byrne, 2003; García-Izquierdo, Sáez y Llor, 2000; Lee y Asforth, 1996; Maslach y Leiter, 1997; Wright y Cropanzano, 1998, Maslach; et al., 2001). También hay evidencias de que la persona que desarrolla burnout puede dar lugar a un efecto de contagio y afianzarse el problema en las relaciones interpersonales en el trabajo (Maslach et al., 2001).

En cuanto a los antecedentes, se suele resaltar la importancia de distintos estresores laborales (Zellars, Perrewé y Hochwarter, 2000) como la sobrecarga de trabajo, el conflicto de rol, la ambigüedad de rol y la falta de autonomía (Lee y Asforth, 1996; Maslach et al., 2001; Schaufeli y Buunk, 2002), y más recientemente el mobbing (García-Izquierdo, Llor, GarcíaIzquierdo, y Ruiz, 2006). Los estresores laborales son considerados como claros antecedentes del burnout, pero cada vez parece más evidente la incidencia de características individuales. La idea fundamental subyacente es que el riesgo de padecer burnout puede diferir no sólo por las situaciones sino también por las variables individuales. En este sentido, se han encontrado evidencias de variabilidad individual frente a los estresores (Moya-Albiol, Serrano, González, Rodríguez-Alarcón y Salvador, 2005), de manera que están ganando peso los modelos que abogan por la interacción entre las variables del entorno laboral y de personalidad (Gil-Monte y Peiró, 1997). Sin embargo, mientras que las características organizativas y del puesto de trabajo han ocupado una buena parte de la investigación del burnout, algunos determinantes individuales han sido tratados someramente. Esto no es nuevo: hace más de veinte años, Kahill (1988) revisó la evidencia empírica y concluyó que la influencia de las características individuales sobre el burnout había sido prácticamente ignorada. Algo similar es lo que sucede con la personalidad, aunque encontremos trabajos pioneros que nos indican que las personas propensas al burnout son más empáticas, obsesivas y susceptibles de sobreidentificarse con los demás (Cherniss, 1980; Pines y Aronson, 1980).

Además, se han analizado propiedades como la personalidad resistente (resiliency), el locus de control, el patrón de conducta tipo A o la ansiedad. Así, se asocia la personalidad resistente (implicación en las actividades diarias, sentido de control sobre las situaciones y apertura a los cambios) a menor burnout (Semmer, 1996; Rowe, 1997; Schaufeli y Ezmann, 1998). En esta línea, Moreno-Jiménez, Morett, Rodríguez, y Morante (2006) muestran que el componente de la personalidad resistente denominado reto (estar abierto a los cambios) amortigua la influencia de los estresores laborales en la ocurrencia del burnout. La personalidad resistente actúa como modulador que reduce la posibilidad de experimentar el desgaste profesional ante la presencia de estresores. Como indica Semmer (1996), las personas con locus de control externo puntúan más alto en burnout que aquellas con locus de control interno. Glass y McKnight (1996), tras una revisión de once estudios, concluyeron que el locus de control externo explica el $10 \%$ de la varianza del agotamieno emocional. También se han encontrado relaciones entre el patrón de conducta tipo A y burnout (Burke, Greenlass y Konarski, 1995; Parker, 1998), y burnout y ansiedad (Cherniss, 1980; Rowe, 1997). El principal problema de estos estudios es que las características de personalidad utilizadas parecen depender más de una elección arbitraria de los investigadores que basadas en un modelo teórico de la personalidad (Bakker, Van der Zee, Lewing y Dollard, 2006). Como señala Salgado (1998a), las personas que presentan un riesgo alto de sufrir burnout se caracterizan por manifestar ansiedad, y bajos niveles de actividad y de control emocional, así como dificultades para delegar y establecer los límites en las relaciones profesionales, lo que conecta con el modelo de personalidad de los cinco factores (Big Five Factors). En la actualidad, existe consenso acerca de la utilidad de este modelo para describir la personalidad cuyo supuesto básico es que cinco factores, cada uno con polos opuestos, son suficientes para describir la personalidad. Tales factores reciben diversas denominaciones dependiendo de los diferentes autores, aunque podemos seguir a Norman (1963): Neuroticismo (N), preocupación, inseguridad, tensión; Extraversión (E), sociabilidad, locuacidad, optimismo; Apertura a la experiencia $(\mathrm{O})$, originalidad, creatividad, independencia; Amigabilidad (A), compasión, confianza, cortesía; 
y Conciencia (C), diligencia, fiabilidad, organización. Varios estudios y meta-análisis llevados a cabo en los últimos años como los de Barrick y Mount (1991), Judge, Heller y Mount (2002), Salgado (1997, 1998b, 2003) y Tett, Jackson y Rothstein (1991), han puesto de manifiesto la capacidad de los Big Five para predecir variables de resultado en las organizaciones (García-Izquierdo, García-Izquierdo y RamosVillagrasa, 2007). Algunas de las dimensiones de personalidad (por ejemplo, Conciencia, Amigabilidad y Neuroticismo) han mostrado que son válidos predictores del desempeño en el puesto y de conductas contraproductivas, tales como robos, problemas de disciplina y absentismo (Ones, Viswesvaran y Schmidt, 1993). Piedmont (1993), siguiendo este modelo de personalidad, en un estudio longitudinal, obtuvo asociaciones significativas en los factores N, E y A con burnout y señaló que $\mathrm{N}$ y A explicaban el $42 \%$ del agotamiento emocional. Sin embargo, Deary et al., (1996) no encontró relaciones entre A y agotamiento emocional. Schaufeli y Ezmann (1998), por su parte, analizaron la contribución de los cinco factores al burnout y concluyeron que $\mathrm{N}$ y $\mathrm{O}$ se asociaban a agotamiento emocional y explicaban un 33\% de la varianza. Esto contradice el resultado de Piedmont (1993) quien no encontró relaciones del agotamiento emocional con O. En resumen, se suele considerar que el factor de personalidad que más se asocia al agotamiento emocional es el de neuroticismo (Deary et al., 1996; Buhler y Land, 2003; Zellars et al. 2000). A la vista de lo anterior, en nuestro estudio planteamos la hipótesis siguiente:

Hipótesis 1: Los cinco factores de personalidad del modelo se asociarán significativamente con el agotamiento emocional, de forma positiva con Neuroticismo y negativamente con los demás.

En los últimos años, la investigación ha centrado su atención en los recursos personales como afrontamiento ante el burnout (Schaufeli y Bakker, 2004). Aunque el burnout es frecuentemente señalado como una respuesta a los factores organizativos, la comprensión del burnout será mayor si avanzamos en el estudio de las variables de personalidad. Los factores del entorno pueden ser relevantes sólo en el grado en que los individuos son capaces de expresar sus niveles de estrés. Algunos autores (McCrae y Costa, 1987; Piedmont, 1993) mostraron que ciertos individuos son capaces de adaptarse adecuadamente a condiciones estresantes y volver a sus niveles iniciales de bienestar, hallazgo que ha recibido un fuerte apoyo en los estudios de la psicología positiva. Este enfoque se centra en las fortalezas humanas que actúan como freno frente a las adversidades y experiencias traumáticas, antes que en los procesos de enfermedad (Seligman y Csikszentmihalyi, 2000). Cuando se aplica al ámbito laboral hablamos de comportamiento organizacional positivo (Positive Organizacional Behavior, POB) (Luthans, 2002). El constructo Resistencia (Resilience), incluido en la $\mathrm{POB}$, engloba el proceso que facilita la superación de las adversidades, salir fortalecido de la experiencia, y desarrollar competencia pese a estar expuesto a altos niveles de estrés psicosocial. Se ha asociado con capacidades personales positivas como la felicidad (Csikszentmihalyi, 1999). También con dimensiones de personalidad: las personas con alta resistencia muestran más estabilidad emocional (Bonanno, Papa y O’Neill, 2001), y son más flexibles a los cambios y más abiertas a nuevas experiencias (Tugade y Fredrickson, 2004) que aquellas con baja resistencia. Por otro lado, recientemente, diversas investigaciones han mostrado relaciones entre resistencia y burnout (Edward, 2005; Howard y Jonson, 2004, Menezes de Lucena, Fernández, Hernández, Ramos, y Contador, 2006).

Sin embargo, la resistencia se ha tratado en la investigación de forma tangencial, quizás por que no existe consenso sobre su carácter estable o contingente. La resistencia puede considerarse un constructo de personalidad (Block, 1961; Menezes de Lucena et al., 2006), aunque otros autores consideran que es más un estado que un rasgo y, por lo tanto, modificable (Connor y Davidson, 2003, Luthans, Vogelgesang y Lester, 2006). Tampoco queda claro si es un constructo de una o varias dimensiones. En este sentido, Luthans et al., (2006) consideran la resistencia como unidimensional y señalan su diferenciación de otros constructos relacionados como esperanza (hope), optimismo (optimism) y confianza (confidence), aunque juntos pueden formar una dimensión de orden superior. Sin embargo (Connor y Davidson, 2003), plantean un modelo multidimensional donde la resistencia se compone de los factores siguientes: competencia personal, confianza, aceptación positiva del cambio, control e influencia espiritual.

Como señalábamos anteriormente, las variables individuales y del entorno forman un proceso de interacción que puede desembocar en altos niveles de tensión y malestar, pero también puede aumentar la capacidad de responder eficazmente o reducir la probabilidad de que las personas desarrollen desajuste psicosocial (Manzano, 2000). Es decir, en la interacción entre variables individuales y del entorno unas personas pueden aumentar sus niveles de estrés mientras que otras no. Por los motivos señalados, consideramos de gran interés estudiar las variables individuales antecedentes, en nuestro caso los cinco factores centrales de personalidad y la resistencia, tomada ésta última como moderadora de la vulnerabilidad del trabajador frente a situaciones de riesgo de estrés laboral crónico (burnout). Por lo tanto, en nuestro trabajo plantemos la siguiente hipótesis:

Hipótesis 2: La resistencia es una variable moderadora entre los cinco factores de personalidad del modelo y el agotamiento emocional. 
En resumen, el propósito de esta investigación es analizar las relaciones entre la personalidad, como característica estable, y la resistencia, como capacidad modificable, con la dimensión agotamiento emocional en una muestra variada de trabajadores.

\section{Método}

\section{Participantes y procedimiento}

Participaron en el estudio 311 trabajadores. En la Tabla 1 se puede apreciar la distribución de los participantes en función de las principales variables sociodemográficas de nuestra investigación: sexo, edad, educación y sector ocupacional. Se observa que el porcentaje de mujeres $(57.24 \%)$ es superior al de hombres $(42.44 \%)$. Respecto a la ocupación, la mayor parte trabaja en el sector servicios $(67.21 \%)$ y el resto se distribuye entre el sector de la construcción $(15.75 \%)$ y la industria (7.72\%). En cuanto a la edad, la media es 31.13 y la desviación típica 11.14 , con una edad mínima de 16 y máxima de 65 (rango de 47). El nivel de estudios se divide en $6.43 \%$ sin estudios, $38.90 \%$ primarios, $13.83 \%$ secundarios, $19.80 \%$ superiores y el $20.26 \%$ que no contesta.

\begin{tabular}{llr} 
Tabla 1. Participantes & \\
\hline Sexo & Mujer & $178(57.24 \%)$ \\
& Hombre & $132(42.44 \%)$ \\
& No contesta & $1(0.32 \%)$ \\
Edad & 21 años o menos & $47(15.11 \%)$ \\
& $21-24$ años & $73(23.47 \%)$ \\
& $25-32$ años & $61(19.61 \%)$ \\
& $33-42$ años & $68(21.87 \%)$ \\
& 43 años o más & $60(19.30 \%)$ \\
Educación & No contesta & $2(0.64 \%)$ \\
& Sin estudios & $20(6.43 \%)$ \\
& Primarios & $121(38.90 \%)$ \\
& Secundarios & $43(13.83 \%)$ \\
& Superiores & $64(20.58 \%)$ \\
& No contesta & $63(20.26 \%)$ \\
Sector & Servicios & $209(67.21 \%)$ \\
& Construcción & $49(15.75 \%)$ \\
& Industria & $24(7.72 \%)$ \\
& No contesta & $29(9.32 \%)$
\end{tabular}

Nota. $N=311$

La recogida de datos contó con el apoyo de distintas organizaciones con las que se contactó previamente durante el año 2007. Se aplicaron 386 cuestionarios de los que 311 fueron válidos (80.57\%). A todos los participantes se les aseguró la confidencialidad y anonimato en sus respuestas y contaron con personal entrenado para responder las dudas que pudieran plantear. En el análisis de datos se utilizó el programa estadísti- co SPSS versión 15.0 realizando análisis descriptivos, de fiabilidad, diferencia de medias, correlaciones entre las variables objeto de interés y regresión lineal múltiple (stepwise). Para minimizar los efectos de la multicolinealidad en los análisis de regresión se transformaron las puntuaciones directas en puntuaciones típicas (Aiken y West, 1991). Se utilizó el tratamiento listwise para los datos perdidos.

\section{Instrumentos}

Las principales variables incluidas en el estudio (burnout, personalidad y resistencia) fueron medidas con los instrumentos que a continuación se describen.

Como medida del burnout utilizamos la escala MBI (Maslach Burnout Inventory) de Maslach, y Jackson (1986) en la versión española de Gil-Monte y Peiró (1999). Esta escala consta de tres dimensiones: agotamiento emocional, despersonalización y realización personal. En nuestro estudio hemos utilizado la escala de agotamiento emocional (9 ítemes), la dimensión más estudiada que refleja el componente más asociado al estrés laboral del instrumento (Maslach, et al. 2001). Un ejemplo de ítem es "Trabajar todo el día es una tensión para mí". Todos los ítems se valoran mediante una escala de cinco puntos que va desde "1" (nunca) hasta "5" (siempre). La puntuación mínima fue 9 y la máxima 42 (rango: 33).

La personalidad fue medida con la versión reducida del Big Five Inventory (BFI) de John, Donahue y Kentle (1991) elaborada por Benet-Martínez y John (1998). Este instrumento fue diseñado simultáneamente en inglés y en español, y consta de 44 ítemes en los que se pide al participante que valore en una escala de respuesta tipo Likert de 5 puntos, en qué medida cada una de las afirmaciones le describe adecuadamente. Mediante este instrumento se miden los cinco factores de personalidad. Neuroticismo $(\mathrm{N})$ consta de 8 ítemes, por ejemplo "Se pone nervioso con facilidad", la puntuación mínima fue de 11 y la máxima de 37 (rango: 26). Extraversión (E) se evalúa mediante 8 ítemes, siendo un ejemplo de los mismos "No teme expresar lo que quiere"; obteniendo una puntuación mínima de 12 y una máxima de 40 (rango: 28). Apertura a la experiencia $(\mathrm{O})$ tiene 10 ítemes de los cuales un ejemplo es "Tiene intereses muy diversos"; con una puntuación mínima de 19 y máxima de 50 (rango: 31). Amigabilidad (A) consta de 9 ítemes y un ejemplo es "Es generoso y ayuda a los demás"; con una puntuación mínima de 20 y una máxima de 45 (rango: 25). Por último, el factor Conciencia (C) consta de 9 ítemes, siendo un ejemplo "Es minucioso en el trabajo", su puntuación mínima fue 20 y la máxima 45 (rango: 25).

Para la medida de la variable resistencia se empleó la escala Connor-Davidson Resilience Scale (CDRISC) elaborada por Connor y Davidson (2003) basándose en los trabajos previos de Kobasa (1979), 
Rutter (1985) y Lyons (1991). Consta de un total de 25 ítemes distribuidos en cinco dimensiones: competencia personal, confianza en los propios instintos, aceptación positiva del cambio, control e influencias espirituales. Hemos adaptado esta escala siguiendo las recomendaciones de la International Test Comission (ITC) (Balluerka, Gorostiaga, AlonsoArbiol, y Aramburu, 2007; Hambleton, 1994; Hambleton y Jong, 2003; Van de Vijver y Hambleton, 1996). Tras los pertinentes análisis estadísticos (ver Tabla 2) que se describen en el apartado de resultados optamos por utilizar un cuestionario de 13 afirmacio-
1", explica un $8.35 \%$ de la varianza y su contenido es similar al componente Competencia personal de la escala original. El segundo componente, que hemos llamado "Resistencia 2" explica el $37.79 \%$ de la varianza y su estructura, aunque semejante al componente "Aceptación positiva del cambio" de Connor y Davidson (2003), incorpora los ítemes que no formaron parte de la primera dimensión. Esto da lugar a un componente referido a la creencia del individuo de ser capaz de conseguir sus objetivos, vencer las adversidades y manejar situaciones complicadas, contenido semejante al de la autoeficacia (Bandura, 1987). Dada

\begin{tabular}{|c|c|c|c|c|c|c|c|}
\hline \multirow[b]{2}{*}{ Item $n^{0}$} & \multirow[b]{2}{*}{ Ítemes en inglés } & \multirow[b]{2}{*}{ Item $n^{0}$} & \multirow[b]{2}{*}{ Ítemes en español (traducción) } & \multicolumn{2}{|c|}{$\begin{array}{c}\text { Connor } \\
\text { y Davidson (2003) } \\
\end{array}$} & \multicolumn{2}{|c|}{$\begin{array}{c}\text { Resultados en } \\
\text { este estudio }\end{array}$} \\
\hline & & & & $\mathrm{CP}$ & $\mathrm{AP}$ & $\mathrm{R} 1$ & $\mathrm{R} 2$ \\
\hline 12 & When things look hopeless, I don't give up & 2 & No me rindo, aunque no haya esperanza & .64 & .21 & .78 & 37 \\
\hline 11 & You can achieve your goals & 3 & Consigo alcanzar mis metas & .62 & .13 & .45 & .64 \\
\hline 25 & Pride in your achievements & 4 & Estoy orgulloso de mis logros & .60 & .15 & .48 & .64 \\
\hline 16 & Not easily discouraged by failure & 8 & No me desanimo fácilmente por los fracasos & .40 & .26 & .61 & 50 \\
\hline 23 & I like challenges & 6 & Me gustan los desafíos & .56 & .01 & .65 & .29 \\
\hline 17 & Think of self as strong person & 7 & Pienso en mí como una persona fuerte & .40 & .13 & .40 & .69 \\
\hline 10 & Best effort no matter what & 5 & Me esfuerzo todo lo que puedo sin importar de lo que se trate & .60 & .17 & .68 & .36 \\
\hline 24 & You work to attain your goals & 1 & Trabajo para conseguir mis metas & .71 & .04 & .60 & .44 \\
\hline 2 & Close and secure relationships & 10 & Tengo relaciones cercanas y estables con los demás & .23 & .54 & .22 & .68 \\
\hline 4 & Can deal with whatever comes & 11 & Puedo manejar cualquier situación posible & .07 & .62 & .46 & .71 \\
\hline 1 & Able to adapt to change & 9 & Sé adaptarme a los cambios & .07 & .76 & .42 & 55 \\
\hline 5 & Past success gives confidence for new challenge & 12 & Mis éxitos pasados me dan fuerza para enfrentarme a nuevos retos & s. .27 & .55 & .37 & .76 \\
\hline 8 & Tend to bounce back after illness or hardship & 13 & Después de una enfermedad o dificultad suelo "volver a la carga" & .34 & .44 & .62 & 52 \\
\hline \multicolumn{4}{|c|}{ Autovalores (Eigenvalues) mayores que uno } & 7.44 & 1.34 & 1.08 & 4.95 \\
\hline \multicolumn{4}{|c|}{ Porcentaje de varianza explicado } & ND & ND & $8.30 \%$ & $38.10 \%$ \\
\hline
\end{tabular}

Nota $. N=311 . \mathrm{CP}=$ Competencia personal. $\mathrm{AC}=$ Aceptación positiva del cambio. $\mathrm{R} 1=$ Resistencia $1 . \mathrm{R} 2=$ Resistencia $2 . \mathrm{ND}=$ No disponible. En cursiva aparecen las cargas relevantes en el segundo factor .

nes, que se valoran según una escala de cinco puntos (desde 1, totalmente incierto, a 5, totalmente cierto) como medida de resistencia total. Un ejemplo de ítem de es "Pienso en mí como una persona fuerte". La puntuación mínima obtenida fue 28 y la máxima 65 , con un rango de 37.

\section{Resultados}

Antes de presentar las relaciones entre las variables, y dada la escasez de estudios sobre resistencia, para estudiar su dimensionalidad se realizó un análisis de componentes principales con rotación oblicua y tanto la prueba KMO (.90) como la de esfericidad de Bartlett $\left(\chi^{2}=1357.94, \mathrm{p}<.001\right)$ mostraron valores adecuados. Como se puede ver en la Tabla 2, los resultados indican la existencia de dos dimensiones, con una composición semejante a la del estudio de Connor y Davidson (2003), aunque las cargas factoriales son algo diferentes.

El primer componente de nuestro estudio, que hemos denominada a efectos operativos "Resistencia la fuerte asociación entre los dos factores $(r=.53$, $\mathrm{p}<0.01)$ y la saturaciones encontradas, decidimos emplear en los análisis posteriores la suma total como el valor de Resistencia, del mismo modo que en el instrumento original.

En la Tabla 3, presentamos los estadísticos descriptivos, la consistencia interna de las escalas y las correlaciones entre las variables del estudio. Estos resultados muestran que las escalas que cuentan con una consistencia interna más alta son la resistencia total $(\alpha=.86)$ y el agotamiento emocional $(\alpha=.83)$; mientras que los factores de personalidad oscilan desde $\alpha=.59$ en el caso de Neuroticismo hasta el $\alpha=.74$ en el caso de Amigabilidad.

Respecto a las asociaciones entre variables, encontramos que se confirma la primera hipótesis, esto es, el Agotamiento emocional se asocia significativamente con todas las variables psicológicas estudiadas y en el sentido esperado según la investigación precedente, esto es, positivamente con Neuroticismo $(r=.36$, $\mathrm{p}<.01$ ) y negativamente con el resto, destacando la asociación con Resistencia ( $r=-.29, \mathrm{p}<.01)$. También resultan destacables las asociaciones encontradas entre 
Tabla 3. Estadísticos descriptivos, correlaciones y fiabilidad

\begin{tabular}{lccccccccccc}
\hline & $M$ & $S D$ & 1 & 2 & 3 & 4 & 5 & 6 & 7 \\
\hline 1. Agotamiento emocional (AE) & 21.01 & 7.27 & $(.83)$ & & & & & & & \\
2. Neuroticismo (N) & 21.81 & 4.91 & $.36^{* *}$ & $(.59)$ & & & & & \\
3. Extraversión (E) & 27.40 & 4.95 & $-.15^{* *}$ & $-.29^{* *}$ & $(.64)$ & & & & \\
4. Apertura a la experiencia (O) & 35.76 & 5.98 & $-.18^{* *}$ & $-.35^{* *}$ & $.40^{* *}$ & $(.74)$ & & \\
5. Amigabilidad (A) & 33.55 & 5.46 & $-.22^{* *}$ & $-.45^{* *}$ & $.24^{* *}$ & $.29^{* *}$ & $(.65)$ & \\
6. Conciencia (C) & 33.08 & 5.11 & $-.19^{* *}$ & $-.43^{* *}$ & $.23^{* *}$ & $.35^{* *}$ & $.52^{* *}$ & $(.66)$ & \\
7. Resistencia & 51.39 & 8.00 & $-.29^{* *}$ & $-.40^{* *}$ & $.45^{* *}$ & $.49^{* *}$ & $.35^{* *}$ & $.50^{* *}$ & $(.86)$ \\
8. Edad & 31.10 & 10.70 & -.00 & $-.23^{* *}$ & .02 & $.13^{* *}$ & $.30^{* *}$ & $.34^{*}$ & $.17^{*}$ & $(1.00)$ \\
\hline
\end{tabular}

Nota. $N=311$. La fiabilidad (alfa de Cronbach) se presenta en la diagonal principal entre paréntesis.

$* p<0.05 . * * p<0.01$

la edad de los participantes con Neuroticismo, con la que se asocia negativamente $(r=-.23, \mathrm{p}<.01)$, y positivamente con Apertura a la experiencia $(r=.13, \mathrm{p}<0.05)$, Amigabilidad $(r=.30, \mathrm{p}<.01)$, Conciencia $(r=.34$, $\mathrm{p}<.01)$ y Resistencia $(r=.17, \mathrm{p}<.05)$. Asimismo, vemos cómo resistencia se asocia significativamente con todas las variables de personalidad, con Agotamiento emocional y con Edad. Las asociaciones son negativas con Agotamiento emocional $(\mathrm{r}=-.29, \mathrm{p}<.001)$ y N $(\mathrm{r}=-$ $.40, \mathrm{p}<.001)$, y positivas con el resto, que van desde la menor $(\mathrm{r}=.17, \mathrm{p}<.05)$ con Edad hasta la mayor con $\mathrm{C}(\mathrm{r}=.50, \mathrm{p}<.001)$, obteniendo con el resto de variables de personalidad, $\mathrm{A}(\mathrm{r}=.35, \mathrm{p}<.001), \mathrm{E}(\mathrm{r}=.45, \mathrm{p}<$ $.001)$ y $\mathrm{O}(\mathrm{r}=.49, \mathrm{p}<.001)$, unas asociaciones destacables.

Del análisis de las interacciones entre las variables, comprobamos que se produce interacción entre Conciencia (C) y Resistencia, confirmando la segunda hipótesis del estudio. Realizamos un análisis de regresión jerárquica múltiple por pasos para determinar los efectos principales de los factores de personalidad sobre burnout. Para minimizar los efectos de la multicolinealidad se realizaron los análisis de regresión con las variables independientes estandarizadas (Aiken y West, 1991). Los resultados muestran la capacidad para predecir el Agotamiento emocional, del factor Neuroticismo en sentido positivo, y de Conciencia y Resistencia en sentido negativo arrojando una $\mathrm{R}^{2}=.17$ $\mathrm{y}$ una $\mathrm{R}^{2}$ ajustada $=.16$.

En la ecuación se establecieron cuatro pasos en los que sucesivamente se fueron añadiendo las diferentes variables independientes estandarizadas (sociodemográficas, personalidad, resistencia e interacción): en primer lugar, las variables sociodemográficas para controlar su posible influencia, seguidamente los cinco factores de personalidad y por último, las interacciones entre las variables independientes (Conciencia y Resistencia). Los resultados, que se muestran en la Tabla 4, presentan una ecuación de regresión en el cuarto paso con tres variables: Neuroticismo $(\beta=.28, \mathrm{p}<.001)$, Resistencia $(\beta=-.17, \mathrm{p}<.05)$ y la interacción Resistencia $\mathrm{x}$ Conciencia" $(\beta=-.10, \mathrm{p}<.05), \mathrm{y}$, que en su conjunto explican el $16 \%$ de la varianza $\left(\mathrm{R}^{2}\right.$ ajustada $\left.=.16\right)$.
Tabla 4. Modelo predictivo para el criterio Agotamiento emocional (incluyendo interacción con Resistencia)

\begin{tabular}{|c|c|c|c|c|c|}
\hline Predictores & $R^{2}$ & $\Delta R^{2}$ & $R^{2}$ aj & $\Delta R^{2} \mathrm{aj}$ & Beta \\
\hline Paso 1: $\quad \mathrm{N}$ & .13 & & .13 & & $.36 * * *$ \\
\hline Paso 2: $\quad \mathrm{N}$ & .15 & .02 & .15 & .02 & $.39 * * *$ \\
\hline Resistencia & & & & & $-.17 * *$ \\
\hline Paso 3: $\quad \mathrm{N}$ & .17 & .02 & .16 & .01 & $.28 * * *$ \\
\hline Resistencia & & & & & $-.17 * *$ \\
\hline Resistencia x C & & & & & $-.10 *$ \\
\hline
\end{tabular}

Como indican Hair, Anderson, Tatham y Black (1999), la interpretación de los coeficientes de regresión es diferente al introducir relaciones moderadas en el modelo. De manera que el efecto moderador nos indica, en nuestro caso, el cambio unitario en el efecto de C cuando la resistencia cambia. Dado que la interacción entre dos factores se produce cuando el efecto de uno de ellos sobre la variable dependiente no es el mismo en todos los niveles del otro factor (Everitt y Howel, 2005). Para estudiar el efecto de interacción procedimos de la siguiente forma: en primer lugar dicotomizamos la variable resistencia en "alta" (una desviación típica o más por encima de la media), "baja" (una desviación típica o más por debajo de la media) y "media" (con valores entre los dos anteriores). Después, calculamos la predicción de C sobre agotamiento emocional para los casos con alta, media y baja resistencia, comprobando su significación estadística. Esto nos permite saber en qué casos se produce el efecto moderador de la resistencia sobre C. Los resultados, representados gráficamente en la Figura 1, indican que la influencia de la variables moderadora se produce únicamente en los casos de resistencia alta $(\beta=-.15, \mathrm{p}<.005)$. Esto supone que el efecto moderador de la resistencia se da únicamente cuando adquiere valores altos, de manera que cuando se da también C alta, el Agotamiento emocional es sustancialmente menor que cuando la resistencia es baja. 
Figura 1. Interacción entre Conciencia y Resistencia en la predicción del Agotamiento emocional

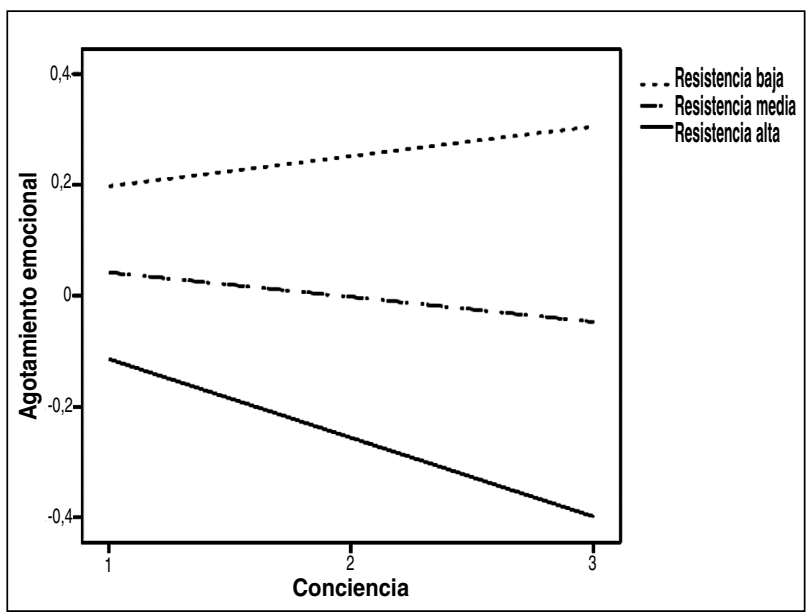

\section{Discusión}

El objetivo del presente estudio era analizar las relaciones entre personalidad y resistencia con agotamiento emocional (burnout) en una muestra de trabajadores. La investigación que relaciona personalidad y burnout, en general, se ha centrado en diferentes características individuales sin fundamentarse en un modelo de personalidad. Nosotros hemos utilizado el modelo de los cinco grandes factores. Del análisis de correlaciones se desprende que el agotamiento emocional se asocia positivamente con el neuroticismo y negativamente con los cuatro factores restantes. Estos resultados confirman la idea de que las personas que puntúan alto en neuroticismo, es decir, que son más inestables emocionalmente, son más vulnerables al estrés psicosocial y es más probable que respondan con agotamiento emocional. Este resultado está en concordancia con lo observado en otras investigaciones (Bakker et al., 2006; Zellars et al., 2000). Sin embargo, no debemos olvidar que la inestabilidad emocional puede actuar como un moderador entre los estresores laborales y los resultados afectivos (Parker, 1998). Por su parte, la Conciencia y la Amigabilidad pueden actuar de forma protectora ante los estresores.

La resistencia es una variable que se ha tenido poco en cuenta en la investigación del burnout por lo que su aportación merece una atención especial. Hemos encontrado correlaciones significativas en sentido negativo entre agotamiento emocional y resistencia. En relación a este hallazgo, el resultado del meta-análisis de Pfennig y Hüsch (1994) (citado en Schaufeli y Buunk, 2002) encuentra relaciones negativas de agotamiento emocional con autoestima y autoevaluación personal de la competencia. También se asocia la resistencia con los factores de personalidad: negativamente con neuroticismo, y positivamente con los demás. Así, aquellas personas que manifiestan agotamiento emocional se caracterizarán por la inestabilidad emocional y una baja resistencia. Los resultados expuestos están en la línea de lo señalado en otras investigaciones. Por ejemplo, Bonanno et al. (2001) encontró que las personas con baja resistencia mostraban menos estabilidad emocional cuando se enfrentan a la adversidad. Tugade y Frederickson (2004) sugirieron que las personas con alta resistencia son más flexibles a los cambios y más abiertas a nuevas experiencias, y con Edward (2005), Howard y Johnson (2004) y Menezes de Lucena et al. (2006) hallaron relaciones negativas entre resistencia y burnout.

El efecto de las variables de personalidad sobre el agotamiento emocional puede estar moderado por distintos recursos personales. Hay pruebas relevantes de que las estrategias de afrontamiento desempeñan una función importante en los resultados del estrés laboral. Con el fin de avanzar más en la clarificación del papel de la resistencia sobre el agotamiento emocional, realizamos un análisis de regresión jerárquico por pasos. Los resultados muestran la capacidad para predecir el agotamiento emocional de distintas variables. En el tercer paso (ver Tabla 4) encontramos que aparecen Neuroticismo, Resistencia y la interacción Resistencia por Conciencia. Por lo tanto, ser inseguro y preocupado (características de neuroticismo), y poseer baja resistencia, así como la interacción con el factor de personalidad Conciencia (ser cuidadoso, fiable y organizado) ambas en sentido negativo, presentan efectos sobre el agotamiento emocional. También hemos constatado el efecto moderador de la resistencia. Cuando la resistencia y la Conciencia son altas, el agotamiento emocional será menor que si la resistencia es baja. Siguiendo a Barrick y Mount (1991), los trabajadores con alta Conciencia (responsables, diligentes) probablemente se vean más afectados por el agotamiento emocional en cuanto a su nivel de rendimiento que aquellos trabajadores que puntúan bajo en Conciencia.

\section{Limitaciones del estudio y líneas de investigación futura}

Vamos a señalar dos limitaciones principales. En primer lugar, los datos recogidos provienen exclusivamente de pruebas de autoinforme. Esta es una práctica usual en los estudios sobre burnout. La utilización de este tipo de instrumentos de medida puede provocar un sesgo en la respuesta de los participantes, exacerbar la varianza común y aumentar artificialmente las correlaciones entre variables (Podsakoff, Mcackenzie, Lee y Podsakoff, 2003; Spector, 2006). En segundo lugar, hemos utilizado un diseño transversal en la recogida de datos. También es cierto que este tipo de diseño es usual en los estudios organizacionales. Las limitaciones de los datos de autoinforme y de los diseños transversales llaman la atención sobre la necesidad de diseños más sofisticados. Una alternativa es emplear diseños longitudinales, los cuales ofrecen indudables ventajas (Taris, 2000; Taris y Kompier, 2003). La utilización de estu- 
dios longitudinales, el uso de información objetiva y subjetiva y la aplicación de técnicas estadísticas que permitan identificar la dirección y magnitud de las relaciones causales entre variables, facilitarán una mejor comprensión de la problemática del burnout.

Las diferencias individuales representan un papel principal al influir en el modo en que los individuos perciben, interpretan y responden a los estresores del entorno laboral. Los resultados encontrados entre personalidad, resistencia y agotamiento emocional, sugieren la pertinencia de continuar realizando estudios en este sentido e incluir variables organizacionales. La investigación en el ámbito del estrés laboral debe avanzar con la elaboración de modelos que tengan en cuenta tanto variables del contexto como individuales que puedan estar relacionadas y moderar la relación entre estresores y consecuencias.
Por último, la resistencia es un buen indicador de la recuperación de los sucesos que afectan de manera traumática a las emociones individuales, y algunos autores señalan que hay evidencias de que la resistencia, antes que un rasgo, puede ser una dimensión que se puede desarrollar (p. ej. Masten y Reed, 2002; Youssef y Luthans, 2005). Ya que no disponemos de datos concluyentes, es recomendable realizar estudios que permitan clarificar este aspecto. Además, los procesos organizacionales actuales van acompañados de exigencias altas de esfuerzo, intensidad, jornadas elevadas y necesidad de adaptación a los cambios, como fuentes de desestabilización emocional, por lo que su inclusión en los modelos de predicción parece pertinente. A la vista de nuestros resultados, la resistencia podría ser uno de los conceptos que se trataran en los programas de prevención del burnout.

\section{Extended Summary}

We are living in an age in which change and turbulence in our surroundings form an integral part of organisational dynamics. Against this new backdrop, where work-related stress is so significant, personal psychological capabilities take on vital importance.

Burnout is a response to chronic work-related stress which consists of emotional exhaustion, depersonalization and a reduction of personal achievements (Maslach, 1993). Initially, Maslach (1986) had identified the so-called 'helping professions' as those where burnout could have greatest presence, but later the concept was applied to other professions (Golembieski, Boudreau, Munzenreider \& Luo, 1996; Scahufeli, Leiter, Maslach \& Jackson, 1996), and it was refined as a difficult situation in work relations, not only or necessarily as a crisis in interpersonal relations, with three aspects being identified, similar to those already quoted. These were: exhaustion, cynicism and low professional efficacy. There is general consensus that emotional exhaustion is the key dimension in burnout syndrome (Shirom, 1989; Lee \& Asforth, 1996; Maslach, Schaufeli \& Leiter, 2001).

One constant in research into burnout has been the search for consequences and causes. Focussing on the causes, we find that work-related stressors are the most important, but the role played by individual characteristics is also becoming increasingly clear. The fundamental underlying idea is that the risk of suffering burnout can vary, not only according to situations, but also according to individual variables, meaning that those models which choose to focus on the interaction between work environment variables and personality are gaining increasing recognition (Gil-Monte \& Peiró, 1997). Over thirty years ago, Kahill (1988) reviewed the empirical evidence and concluded that the influence of individual characteristics on burnout had been ignored. This is the case with personality. Nevertheless, pioneering work can be found which indicates that those individuals more prone to burnout are more empathetic, more obsessive, and more likely to overidentify with other people (Cherniss, 1980; Pines \& Aronson, 1980). In addition, properties such as the resistant personality, the locus of control, type A behaviour patterns and anxiety have all been analysed. Thus, resistant personalities (showing commitment in daily activities, having a sense of control over situations and being open to change) are associated with lower burnout (Semmer, 1996; Rowe, 1997; Schaufeli \& Ezmann, 1998). In a similar way, Moreno-Jiménez, et al. (2006) show that the component of resistant personality referred to as 'challenge' (being open to change) offsets the influence of work-related stressors in the incidence of burnout. Resistant personalities act as a moderator which reduces the chances of becoming worn down professionally when faced with stressors.

The principal problem of these studies is that the personality characteristics that are used appear to depend more on an arbitrary choice made by the researchers than being based on any theoretical personality model (Bakker, Van der Zee, Lewing \& Dollard, 2006). However, there is now consensus that the 'Big Five Factors' model is adequate for describing personality. Various studies and metaanalyses undertaken in recent years, such as those by Barrick \& Mount (1991), Salgado (1997, 1998, 2003), Tett, Jackson and Rothstein (1991), and Judge, Heller \& Mount (2002), have shown this model's ability to predict result variables in organisations (García-Izquierdo, García- 
Izquierdo \& Ramos-Villagrasa, 2007), and it is generally considered that the personality factor most associated with emotional exhaustion is neuroticism (Mills \& Huebner, 1998; Zellars et al. 2000). In our study we shall consider the following hypothesis:

Hypothesis 1: The model's five personality factors will show significant association with emotional exhaustion, positively in the case of Neuroticism and negatively in the others.

In recent years, research has centred its attention on the role of personal resources as a way of coping when faced with burnout (Schaufeli \& Bakker, 2004). Some authors (Costa, McCrae \& Zonderman, 1987; McCrae $\&$ Costa, 1987; Piedmont, 1993) have shown that certain individuals are able to adapt adequately to stressful conditions and return to their initial levels of wellbeing, a phenomenon which studies based on positive psychology have moved forward significantly. When this is applied to the workplace, we may talk about 'positive organisational behaviour' (POB) (Luthans, 2002). The 'Resiliency' construct, which is included in $\mathrm{POB}$, encompasses the process by which an individual is able to overcome difficulties and come out of the experience stronger, to develop competency despite being exposed to high levels of psychosocial stress. The construct has even been associated with positive personal capacities such as happiness (Csikszentmihalyi, 1999), sense of coherence (Lindström, 2003), and well-being at work (Beddington et al. 2008). Similarly with personality dimensions: people with higher resilience show themselves to be more emotionally stable (Bonanno, Papa \& O'Neill, 2001), and are more flexible to change and more open to new experiences (Tugade \& Fredrickson, 2004) than those with low resilience. In addition, various investigations have recently shown relationships between resilience and burnout (Edward, 2005; Howard \& Jonson, 2004, Menezes de Lucena et al., 2006). Nevertheless, resilience has only been dealt with in research at a tangent, perhaps because there has been no consensus about its contingent or stable factor.

As we indicated earlier, in the interaction between individual variables and the surroundings, certain individuals may have increased stress levels, while others do not. For the reasons already explained, we consider the study of individual background variables to be of great interest. In this particular case these variables are the five central personality factors and resilience, with resilience being a moderator of the worker's vulnerability in situations of chronic work-related stress (burnout). Therefore, in this investigation we shall consider the following hypothesis:

Hypothesis 2: resilience is a moderating variable between the model's five personality factors and emotional exhaustion.

\section{Method}

\section{Participants and procedure}

311 workers participated in the study. As can be seen in Table 1, the percentage of women (57.24\%) was greater than that of men $(42.44 \%)$. Regarding occupation, the majority work in the services sector $(67.21 \%)$, and the rest are distributed between the construction sector $(15.75 \%)$ and Industry $(7.72 \%)$. In terms of age, the average is 31.13 and the standard deviation 11.14, with a minimum age of 16 and maximum of 65 (range of 47). The level of education is divided into $6.43 \%$ of participants who have no formal qualifications, $38.90 \%$ with Primary education, $13.83 \%$ with Secondary education, $19.80 \%$ with Higher education and $20.26 \%$ not replying.

The data were collected in the year 2007 and 386 questionnaires were administered of which 311 were valid $(80.57 \%)$. All participants were given assurances as to the confidentiality and anonymity of their answers, and had trained staff available to answer any queries that they might have. Descriptive analyses of reliability, of difference of averages, of correlations between the variables of interest and multiple linear regression ('stepwise') were all carried out. In order to minimise the effects of multicolinearity in the regression analyses, the scores were converted into typical scores (Aiken y West, 1991). For missing data, 'listwise' treatment was used.

\section{Instruments}

The main variables included in the study were measured using the instruments described below.

To measure emotional exhaustion, Maslach, and Jackson's MBI (Maslach Burnout Inventory) (1996) scale was used in its Spanish version by Gil-Monte \& Peiró (1999). This contains 9 items, and includes the component work stress (Maslach, Schaufeli \& Leiter, 2001).

Personality was measured with a reduced version of John, Donhaue y Kentle's 'Big Five Inventory' (BFI) (1991) produced by Benet-Martínez y John (1998). This instrument was designed simultaneously in English and Spanish and measures the five personality factors. Neuroticism (N) and Extraversion each consist of 8 items; Openness to Experience $(\mathrm{O})$ has 10 items; Agreeableness (A) consists of 9 items; and, finally, the Conscientiousness factor (C) consists of 9 items.

For the measuring of the resilience variable, the Connor-Davidson Resilience Scale (CD-RISC) developed by Connor y Davidson (2003) was used. The subscales of Personal Competence (7 items) and Positive Acceptance of Change (6 items) were used, and the sum up was used as an estimate of resilience. 


\section{Results}

The descriptive statistics, reliability of scales and correlations between the variables being studied were all obtained. These results, which are presented in Table 3, show that in general the instruments used were adequate for the purposes of research.

With regard to the associations between variables, emotional exhaustion was found to be significantly associated with all of the psychological variables studied, and in the same way as previous research had suggested. Also worthy of note were the associations found with the age of participants, which associates negatively with neuroticism $(r=-0.36, \mathrm{p}<.01)$, and positively with $\mathrm{O}(r=.13, \mathrm{p}<0.05), \mathrm{A}(r=.30, \mathrm{p}<.01), \mathrm{C}(r=.34$, $\mathrm{p}<.01)$ and Resilience $(r=.17, \mathrm{p}<.05)$.

Next, a stepwise hierarchical multiple regression analysis was carried out to determine the main effects of the personality factors on emotional exhaustion. To minimise the effects of multicolinearity, the regression analyses were conducted with standardised independent variables (Aiken \& West, 1991). The results show the possibility of predicting emotional exhaustion from Neuroticism, in a positive direction, and Conscientiousness and Resilience in the opposite sense, giving an $\mathrm{R}^{2}=.17$ and an $\mathrm{R}^{2}$ Adjusted $=.16$.

Subsequently, and with the aim of finding the predictive value of the personality factors and resiliency for emotional exhaustion, and the possible moderating effect produced by the interaction of the personality factors, a multiple hierarchical regression analysis was undertaken. It was found that an interaction takes place between Conscientiousness (C) and Resiliency. Subsequently, a hierarchical regression analysis was carried out with the aim of testing the main effects and the interaction of the personality factors on emotional exhaustion. In the equation, three steps were created, in which the different standardised independent variables (sociodemographic variables, variables of personality, of resilience and interaction) were successively added. The results, presented in Table 4 , show a regression equation with three terms: Neuroticism $(\beta=.28, \mathrm{p}<.001)$, Resilience $(\beta=-.17, \mathrm{p}<.05)$, and the Resilience $\mathrm{x}$ Conscientiousness interaction $(\beta=-.10, \mathrm{p}<.05)$, thus explaining $16 \%$ of the variance $\left(\mathrm{R}^{2}\right.$ adjusted $\left.=.16\right)$.

Given that the interaction between the factors occurs when the effect of one of them on the dependent variable is not the same at all levels of the other factor (Everitt and Howel, 2005), the differences between participants were analysed with both low and high resilience. The results, shown graphically in Figure 2, indicate that moderation takes place in cases of high resistance $(\beta=-.15, \mathrm{p}<.05)$.

\section{Discussion}

The objective of this study was to analyse the rela- tionships that personality and resilience have with emotional exhaustion in a sample of workers. As anticipated by previous studies, significant associations were found between the selected variables. The significance of the associations found increases when we consider the scarcity of studies which deal with this issue. Thus, the significance of the personality factors in the fundamental dimension of burnout becomes clear.

Differences in emotional exhaustion were found according to sex, where women scored more highly in this variable than men. However, it is difficult to interpret the differences in emotional exhaustion by sex as they can become confused by a whole series of other closely linked variables, such as profession, position held, role stress and so on.

The big five factors model was used, and from the analysis of correlations it can be concluded that Neuroticism associates positively with emotional exhaustion and negatively with the other factors. This confirms the idea that individuals who score highly in neuroticism, who are more emotionally unstable, are more vulnerable to psychosocial stress. This result is in line with the observations of other research (Zellars $e t$ al., 2000; Bakker et al., 2006).

Resilience is one variable which has been considered very little in research, and for this reason its contribution deserves particular attention. Significant correlations were found between emotional exhaustion and resilience. Resilience is a good indicator of recovery from events which traumatically effect the individual's emotions. In addition, current organisational processes involve high demands for effort, intensity, number of working days and the need for adaptation to change, all of which act as sources of emotional destabilization, meaning that their inclusion in models would seem pertinent.

In this study we have found that resilience acts as a moderator between personality and emotional exhaustion. These results suggest that being insecure and worried ( $\mathrm{N}$ characteristics), and suspicious and ungenerous (A characteristics in its negative pole) show direct effects on emotional exhaustion. Resilience is found in the interaction with the $\mathrm{C}$ personality factor (in a negative direction). Therefore, we believe it to be essential that in subsequent investigations resiliency variables are included as a relevant predictor in the area of burnout and stress.

\section{Referencias}

Aiken, L. S., y West, S. G. (1991). Multiple regression: Testing and interpreting interactions. Newbury Park, CA: Sage.

Bakker, A., Van der Zee, K., Lewig, K., y Dollard, M. (2006). The relationship between the big five personality factors and burnout: a study among volunteer counsellors. The Journal of Social Psychology, 146, 31-50. 
Balluerka, N., Gorostiaga, A., Alonso-Arbiol, I., y Aramburu, M. (2007). La adaptación de instrumentos de medida de unas culturas a otras: una perspectiva práctica. Psicothema, 19, 124-133.

Bandura, A. (1977). Self-efficacy: Toward a unifying theory of behavioral change. Psychological Review, 84, 191-215.

Bandura, A. (1987/[1986]). Pensamiento y acción. Barcelona: Martínez Roca.

Barrick, M.R., y Mount, M.K. (1991). The big five personality dimensions and job performance: A meta- analysis. Personnel Psychology, 44, 1-26.

Beddington, J., Cooper, C.L., Field, J., Goswami, U., Huppert, F. A., Jenkins, R., Jones, H.S., Kirkwood, T.B.L., Sahakian, B.J., y Thomas, S.M. (2008). The mental wealth of nations. Nature, 455, 1057-1060.

Benet-Martínez, V., y John, O.P. (1998). Los Cinco Grandes across cultures and ethnic groups: multitrait multimethod analyses of the Big Five in Spanish and English. Journal of Personality and Social Psychology, 75, 729-750.

Block, J. (1961). Ego-identity, role variability, and adjustment. Journal of Consulting and Clinical Psychology, 25, 392-397.

Bonnano, G.A., Papa, A., y O' Neill, K. (2001). Loss and human resilience. Applied Preventive Psychology, 10, 193-206.

Burke, R. J., y Greenglass, E. (1996). Work stress, social support, psychological burnout and emotional and physical well-being among teachers. Psychology, Health and Medicine, 1, 193-205.

Burke, R. J., Greenglass, E., y Konarski, R (1995). Coping, work demands and psychological burnout among teachers. Journal of Health and Human Services Administration, 18, 90-103.

Cherniss, C. (1980). Staff Burnout: Job Stress in the human services. Beverly Hills: SAGE

Connor, K.M., y Davidson, M.D. (2003). Development of a new resilience scale: the Connor-Davidson resilience scale (CD-RISC). Depression and Anxiety, 18, 76-82.

Cropanzano, R., Ruppe, D.E. y Byrne, Z.S. (2003). The relationship of emotional exhaustion to work attitudes, job performance, and organizational citizenship behaviors. Journal of Applied Psychology, 88, 160-169.

Csikszentmihalyi, M. (1999). If we are rich, why aren't we happy. American Psychologist, 54, 76-82.

Edward, K.L. (2005) The phenomenon of resilience in crisis care mental health clinicians. International Journal of Mental Health Nursing, 14(2), 142-148.

Everitt, B.S., y Howell, D.C. (2005). Encyclopedia of statistics in behavioral science (vol.2, pp.929-933). Chichester, Sussex: Wiley.

García-Izquierdo, A.L., García-Izquierdo, M., y RamosVillagrasa, P.J. (2007). Inteligencia Emocional y Autoeficacia. Aportaciones para la selección de personal. Anales de Psicología, 23, 231-239.

García-Izquierdo, A.L., y Ramos-Villagrasa, P.J. (2009). Calidad de vida laboral: una perspectiva psicosocial. En A. M. Guillén, R. Gutiérrez y S. Begega (Eds), Calidad del trabajo en la Unión Europea. Concepto, Tensiones, Dimensiones. Navarra: Aranzadi-Thomson Civitas.

García-Izquierdo, M., Sáez, C., y Llor, B. (2000). Burnout, satisfacción laboral y bienestar en personal sanitario de salud mental. Revista de Psicología del Trabajo y de las Organizaciones, 16, 215-228.

García-Izquierdo, M., Llor, B., García- Izquierdo, A.L., y Ruiz, J.A. (2006). Bienestar psicológico y mobbing en una muestra de profesionales de los sectores educativo y sanitario. Revista de Psicología del Trabajo y de las Organizaciones, 22, 381-396.

Gil-Monte, P. y Peiró, J. M. (1999). Validez factorial del Maslach Burnout Inventory en una muestra multiocupacional. Psicothema, 11(3), 679-689.

Glass, D., y McKnight, D. (1996). Perception control, depressive symptomatology and professional burnout. Psychological Health, 11, 23-48.

Golembiewski, R. T., Boudreau, R. A., Munzenrider, R. F. y Luo, H. (1996). Global Burnout: A world Wide Pandemic Explored by the Phases Model. Greenwich: JAI Press.

Hair, J.F., Anderson, R.E., Tatham, R.L., y Black, W. (1999). Análisis multivariante. Madrid: Prentice Hall.

Hambleton, R.K. (1994). Guidelines for adapting educational and psychological tests: A progress report. European Journal of Psychological Assessment, 10, 229-240.

Hambleton, R.K., y Jong, J.H. (2003). Advances in translating and adapting educational and psychological tests. Language Testing, 20, 127-134.

Howard, S. y Johnson, B. (2004). Resalient teachers: resisting stress and burnout. Social Psychology of Education, 7, 399-420.

John, O.P., Donahue, E.M., y Kentle, R.L. (1991). The "Big Five" Inventory - Versions $4 a$ and 54. Berkeley, CA: University of California.

Judge, T. A., Heller, D., y Mount, M. K. (2002). Five-Factor model of personality and job satisfaction: A meta-analysis. Journal of Applied Psychology, 87, 530-541.

Kahill, S. (1988). Symptoms of professional burnout: a review of empirical evidence. Canadian Psychology, 29, 284-297.

Kobasa, S. (1979). Stressful life events, personality, and health: An inquiry into hardiness. Journal of Personality and Social Psychology, 37, 1-11.

Lee, R. T., y Ashforth, B. E. (1996). A meta-analytic examination of the correlates of the three dimensions of job burnout. Journal of Applied Psychology, 81(2), 123-133.

Luthans, F. (2002). Positive organizational behavior: Developing and managing psychological strengths. Academy of Management Executive, 16, 57-72.

Luthans, F., y Youssef, C. M. (2004). Human, social, and now positive psychological capital management: Investing in people for competitive advantage. Organizational Dynamics, 33, 1-22.

Luthans, F., Vogelgesang, R., y Lester, P. B. (2006). Developing the psychological capital of resiliency. Human Resource Development Review, 5, 25-44.

Lyons, J. (1991). Strategies for assessing the potential for positive adjustment following trauma. Journal of 
Traumatic Stress, 4, 93-111.

Manzano, G. (2000). Bienestar subjetivo de los cuidadores formales de Alzheimer: relaciones con el burnout, engagement y estrategias de afrontamiento. Ansiedad y Estrés, 8, 225-244.

Maslach, C. (1982). Burnout: The cost of caring. Englewood Cliffs, NJ: Prentice Hall. 1

Maslach, C. (1993). Burnout: a multidimensional perspective. En W.B. Schaufeli, C. Maslach y T. Marek (Eds.) Professional Burnout: Recent Developments in Theory and Research (pp. 19-32). Washington: Taylor y Francis.

Maslach, C., y Leiter, M. P. (1997). The Truth About Burnout. San Francisco: Jossey-Bass.

Maslach, C., Schaufeli, W., y Leiter, M. P. (2001). Job Burnout. Annual Review of Psychology, 52, 397-422.

Masten, A.S. y Reed, M. (2002). Resilience in development. En C.R. Snyder y S. Lopez (Eds.), Handbook of positive psychology (pp. 74-88). Oxford: Oxford University Press.

McCrae, R. R., y Costa, P. T. (1987). Validation on the Fivefactor model $f$ of personality across instruments and observers. Journal of Personality and Social Psychology, 52, 81-90.

Menezes de Lucena, V., Fernández, B., Hernández, L., Ramos, F., y Contador, I. (2006). Resiliencia y el modelo de Burnout-Engagement en cuidadores formales de ancianos. Psicothema, 18, 791-796.

Moreno-Jiménez, B., Morett, N., Rodríguez, A., Morante, M. E. (2006). La personalidad resistente como una variable moduladora del síndrome de burnout en una muestra de bomberos. Psicothema, 18, 413-418.

Moya-Albiol, L., Serrano, M. A., González, E., RodríguezAlarcón, G., y Salvador, A. (2005). Respuesta psicofisiológica de estrés en una jornada laboral. Psicothema, 17, 205-211.

Norman, W.T. (1963). Toward an adequate taxonomy of personality attributes: replicated factors structure in peer nomination personality ratings. Journal of Abnormal and Social Psychology, 66, 574-583.

Ones, D. S. Viswesvaran, C. y Schmidt, F. L. (1993). Comprehensive meta-analysis of integrity test validities: Findings and implications for personnel selection and theories of job performance. Journal of Applied Psychology, 78, 679-703.

Parker, K. (1998) Estrés, trabajo y salud: características laborales, contexto ocupacional y diferencias individuales. En J. Buendía (Ed.). Estrés laboral y salud (pp. 79117). Madrid: Biblioteca Nueva.

Piedmont, R. L. (1993). A Longitudinal Analysis of Burnout in the Health Care Setting: The Role of Personal Dispositions. Journal of Personality Assessment, 61(3), 457-473.

Pines, A., y Aronson, E. (1988). Career burnout: causes and cures. Nueva York: The Free Press.

Podsakoff, P.M., MacKenzie, S.B., Lee, Y. y Podsakoff, N.P. (2003). Common method biases in behavioural research: A critical review of the literature and recommended remedies. Journal of Applied Psychology, 88, 879-903.

Rowe, M. M. (1997). Hardiness, Stress, Temperament,
Coping, and Burnout in Health. American Journal of Health Behavior, 21, 163-171.

Rutter, M.(1985). Resilience in the face of adversity: protective factors and resistance to psychiatric disorders. British Journal of Psychiatry, 147, 598-611.

Salgado, J. (1997). The Five Factor Model of personality and job performance in the European community. Journal of Applied Psychology, 82: 30-43.

Salgado, J. (1998a). Burnout, Personality and Self-Perceived Job Performance. Paper presented at the XXIV Congress of the International Association of Applied Psychology (IAAP), San Francisco (USA), 9-14 August. Paper presented at the XXIV Congress of the International Association of Applied Psychology (IAAP), San Francisco (USA) 9-14 August.

Salgado, J. F. (1998b). Big Five Personality Dimensions and Job Performance in Army and Civil Occupations: A European Perspective. Human Performance, 11, 271288.

Salgado, J.F. (2003). Predicting job performance using FFM and non-FFM personality measures. Journal of Occupational and Organizational Psychology, 76, 323-346.

Schaufeli, W. B., y Bakker, A. B. (2004). Job demands, job resources, and their relationship with burnout and engagement: A multi-sample study. Journal of Organizational Behavior, 25, 293-315.

Schaufeli, W. B., y Buunk, B. P. (2002). Burnout: An overview of 25 years of research and theorizing. En M. J. Schabracq, J. A. M. Winnubst, y C. L. Cooper (Eds.), Handbook of work and health psychology, 383-215. Chichester: Wiley.

Schaufeli, W.B., y Ezmann, D. (1998). The Burnout companion to study and research: A critical analysis. Londres: Taylor \& Francis.

Schaufeli, W. B., Leiter, M. P., Maslach, C. y Jackson, S. E. (1996). Maslach Burnout Inventory-General Survey. En C. Maslach, S. E. Jackson y M. P. Leiter: The Maslach Burnout Inventory-Test Manual ( $3^{\mathrm{a}}$ ed.). Palo Alto, CA: Consulting Psychologists Press.

Schutte, N., Toppinen, S., Kalimo, R., y Schaufeli, W. B. (2000). The factorial validity of the Maslach Burnout Inventory-General Survey (MBI-GS) across groups and nations. Journal of Occupational and Organizational Psychology, 73, 53-67.

Seligman, M., y Csikszentmihalyi, M. (2000). Positive psychology: An introduction. American Psychologist, 55, 5-14.

Semmer, N. (1996). Individual differences, work stress, and health. En M. J. Schabracq, J. A. M. Winnubst, y C. L. Cooper (Eds.), Handbook of work and health psychology, 51-86. Chichester: Wiley.

Shirom, A. (1989). Burnout in work organizations. En C. L. Cooper e I. Robertson (Eds.), International Review of Industrial and Organizational Psychology , 25-48. Nueva York: Wiley.

Sparks, K., Faragher, B. y Cooper, C.L. (2001). Well-being and occupational health in the $21^{\text {st }}$ century workplace. Journal of Occupational and Organizational Psychology, 74, 489-509. 
Spector, P.E. (2006). Method variance in organizational research: Truth or urban legend? Organizational Research Methods, 9, 221-232.

Taris, T.W. (2000). A primer in longitudinal data analysis. Londres: Sage.

Taris, T. W. y Kompier, M. (2003). Challenges of longitudinal designs in occupational health psychology. Scandinavian Journal of Work, Environment and Health, 29, 1-4.

Tett, R., Jackson, D., y Rothstein, M. (1991). Personality measures as predictors of job performance: A meta-analytic review. Personnel Psychology, 703-742.

Tugade, M.M. y Frerickson, B.L. (2004). Resilient individuals use positive emotions to bounce back from negative emotional experiences. Journal of Personality and Social Psychology, 86, 320-333.
Van de Vijver, F. y Hambleton, R.K. (1996). Translating tests: some practical guidelines. European Psychologist, 2, 89-99.

Wright, T., y Cropanzano, R. (1998). Emotional exhaustion as a predictor of job performance and voluntary turnover. Journal of Applied Psychology, 83, 486-493.

Youssef, C.M. y Luthans, F. (2005). Resiliency development of organizations, leaders and employees: Multi-level theory building for sustained performance. En W. Gardner, B.J. Avolio, y F.O. Walumbwa (Eds.), Authentic leadership theory and practice. Origins, effects, and development (pp. 303-343). Oxford: Elsevier.

Zellars, K. L., Perrewé, P. L., y Hochwarter, W. A. (2000). Burnout in health care: The role of the five factors of personality. Journal of Applied Social Psychology, 30, 1570-1598.

Manuscrito Recibido: 22/04/2009

Revisión Recibida: 10/07/2009

Aceptado: 17/07/2009 